\title{
Transport of Small Anionic and Neutral Solutes through Chitosan Membranes: Dependence on Cross-Linking and Chelation of Divalent Cations
}

\author{
Ricardo M. P. da Silva, ${ }^{,+, \ddagger}$ Sofia G. Caridade, ${ }^{\dagger, \ddagger}$ Julio San Román, ${ }^{\S}$ João F. Mano, ${ }^{\dagger, \ddagger}$ and \\ Rui L. Reis ${ }^{\dagger, \ddagger}$ \\ 3B's Research Group-Biomaterials, Biodegradables and Biomimetics, Department of Polymer \\ Engineering, University of Minho, Campus de Gualtar, 4710-057 Braga, Portugal, IBB-Institute for \\ Biotechnology and Bioengineering, PT Government Associated Laboratory, Braga, Portugal, and \\ Department of Biomaterials, Institute of Polymer Science and Technology, CSIC, Juan de la Cierva 3, \\ 28006 Madrid, Spain
}

Received February 14, 2008; Revised Manuscript Received June 7, 2008

\begin{abstract}
Chitosan membranes were prepared by solvent casting and cross-linked with glutaraldehyde at several ratios under homogeneous conditions. The cross-linking degree, varying from 0 to $20 \%$, is defined as the ratio between the total aldehyde groups and the amine groups of chitosan. Permeability experiments were conducted using a side-by-side diffusion cell to determine the flux of small molecules of similar size but with different chemical moieties, either ionized (benzoic acid, salicylic acid, and phthalic acid) or neutral (2-phenylethanol) at physiological $\mathrm{pH}$. The permeability of the different model molecules revealed to be dependent on the affinity of those structurally similar molecules to chitosan. The permeability of the salicylate anion was significantly enhanced by the presence of metal cations commonly present in biological fluids, such as calcium and magnesium, but remained unchanged for the neutral 2-phenylethanol. This effect could be explained by the chelation of metal cations on the amine groups of chitosan, which increased the partition coefficient. The cross-linking degree was also correlated with the permeability and partition coefficient. The change in the permeation properties of chitosan to anionic solutes in the presence of these metallic cations is an important result and should be taken into consideration when trying to make in vitro predictions of the drug release from chitosan-based controlled release systems.
\end{abstract}

\section{Introduction}

Chitosan has been proposed for use in a large number of applications in the biomedical field, ${ }^{1-6}$ such as controlled drug release $^{1,2}$ and biomaterial ${ }^{3,4}$ and tissue engineering. ${ }^{5,6}$ Survival of ex vivo constructed tissues after transplantation is often limited by insufficient oxygen and nutrient supply. Strategies aiming at the improvement of neovascularization of engineered tissues are considered key issues. ${ }^{7,8}$ Scaffolds can work as an additional route for the elimination of excreted toxic products and for the supply of nutrients, if those molecules can permeate through the polymeric support. Although this would not constitute a truly alternative strategy for the improvement of neovascularization of engineered tissues, it would help with survival of tissue engineered transplants.

Both in tissue engineering and in drug delivery strategies the knowledge and control of the permeability of chitosan-based materials is very important. On the other hand, the well-known ability of chitosan to complex with divalent cations ${ }^{9-12}$ can interfere with the permeability of anionic molecules through chitosan materials in physiological medium. In fact, human blood plasma possesses typical $\mathrm{Ca}^{2+}$ and $\mathrm{Mg}^{2+}$ concentrations of 2.5 and $1.5 \mathrm{mM}$, respectively. ${ }^{13}$ To our knowledge, until now, the studies on the permeability of chitosan to several model drugs and solutes have ignored this hypothesis. In the present

* To whom correspondence should be addressed. E-mail: ricardosilva@ dep.uminho.pt.

University of Minho.

$\doteqdot$ Institute for Biotechnology and Bioengineering.

$\S$ Institute of Polymer Science and Technology. study, we tested the influence of the binding ability of divalent cations on permeation of small anionic solutes and its dependence on cross-linking degree.

Finally, the importance of studying the influence of ionic species able to complex with polyelectrolytes on the permeability is not limited to the chitosan-based permeable system that is presented as a case study. In fact, there is a broad list of other polyelectrolytes, both polysaccharides ${ }^{14-16}$ and synthetic polymers, ${ }^{17-19}$ which are able to form complexes with multivalent ions. Polyelectrolyte hydrogels are important systems for controlled drug release applications due to their ( $\mathrm{pH}$ and electric) stimuli-responsive nature. $^{20-22}$

\section{Materials and Methods}

Purification and Characterization of Chitosan. Chitosan (CTS) raw material obtained from crab shells was purchased from SigmaAldrich (U.S.A.) and purified by reprecipitation. First, the chitosan was dissolved in an aqueous acetic acid solution (1\% w/v) at $\sim 1 \%(\mathrm{w} / \mathrm{v})$. The solution was filtered through a Whatman ashless filter paper (20-25 $\mu \mathrm{m})$ to remove the insoluble material and produce a clear solution. This solution was precipitated by adding a $\mathrm{NaOH}$ solution (final $\mathrm{pH} \sim$ 8), forming a white gel, which was sieved to remove the exuded liquid and thoroughly rinsed with distilled water until no changes in the $\mathrm{pH}$ were detected. The chitosan gel was further washed/dehydrated with ethanol, freeze-dried, ground to powder, and dried at $60{ }^{\circ} \mathrm{C}$ overnight. All other reagents were used without further purification.

The chitosan average molecular weight was found to be $7.9 \times 10^{5}$ $\mathrm{g} / \mathrm{mol}$ by viscometry in $\mathrm{CH}_{3} \mathrm{COOH} 0.5 \mathrm{M} / \mathrm{NaCH}_{3} \mathrm{COO} 0.2 \mathrm{M}$ (intrinsic viscosity $10.6 \mathrm{dl} / \mathrm{g})$, according to the Mark-Houwink theory $(k=3.5$ $\times 10^{-4} ; a=0.76$ ). ${ }^{23}$ The degree of $N$-acetylation (DA) was found to 
be $6.7 \%$, determined by means of first derivative UV spectrophotometry, using both glucosamine (GluN) and $N$-acetylglucosamine (GluNAc) standards for calibration. ${ }^{24}$

Preparation of Chitosan Membranes by Solvent Casting. The chitosan solution was prepared by dissolving chitosan (1 wt \%) in 1 wt $\%$ acetic acid solution. Glutaraldehyde (GA) solutions at concentrations ranging from $0.1 \mathrm{M}$ to $5 \times 10^{-4} \mathrm{M}$ were prepared. The amount of chitosan amine groups $\left(\mathrm{NH}_{2}\right.$; GluN units) can be determined using the following expression

$$
n_{(\mathrm{GluN})}=\frac{m_{(\mathrm{CTS})}}{\left[M_{\mathrm{g}}+\frac{1-\mathrm{DD}}{\mathrm{DD}} M_{\mathrm{a}}\right]}
$$

where $M_{\mathrm{a}}=203 \mathrm{~g} / \mathrm{mol}$ and $M_{\mathrm{g}}=161 \mathrm{~g} / \mathrm{mol}$ are the molecular weights of the GluNAc and GluN units within the copolymer, $m_{\text {(CTS) }}$ is dry weight of chitosan in grams and $n_{\text {(GluN) }}$ is the molar amount of amine groups in that weight of chitosan.

Then, defining the cross-linking degree $(x)$ as the percentage of aldehyde (CHO) groups with respect to the initial free $\mathrm{NH}_{2}$ groups $\left(\mathrm{CHO} / \mathrm{NH}_{2}\right.$ ratio), we can write

$$
x(\%)=\frac{n_{(\mathrm{CHO})}}{n_{\mathrm{NH}_{2}}} \times 100=\frac{2 n_{(\mathrm{GA})}}{n_{(\mathrm{GluN})}} \times 100
$$

and it follows that

$$
x(\%)=\frac{2 V_{(\mathrm{GA})} C_{(\mathrm{GA})} \times\left(M_{\mathrm{g}}+\frac{1-\mathrm{DD}}{\mathrm{DD}} M_{\mathrm{a}}\right)}{m_{(\mathrm{CTS})}} \times 100
$$

where $V_{(\mathrm{GA})}$ and $C_{(\mathrm{GA})}$ are, respectively, the volume and concentration of the glutaraldehyde solutions. Actually, the cross-linking degree defined by eq 2 is the reagents feed ratio because the real cross-linking efficiency depends upon the chemical conversion and on the occurrence of other parallel reactions, which can form either any or longer crosslinks. This terminology is used for convenience because, in a practical point of view, the determination of the real chemical efficiency is not feasible.

The glutaraldehyde solution volume added to a certain amount of the previous chitosan solution was kept constant. The several crosslinking degrees were obtained by only changing the concentration of glutaraldehyde, according to eq 3 . In this way, the reaction volume and polymer concentration were kept constant for all the samples, with only the molar amount of glutaraldehyde added varing. The glutaraldehyde solutions were added dropwise over a period of 5 min under gentle stirring and the resultant solutions were left quiescent for about $1 \mathrm{~h}$ to remove any air bubbles formed. Noncross-linked chitosan membranes (CTS00) were prepared in the same way, but no glutaraldehyde solution was added. Solutions were poured into Petri dishes and dried at room temperature. The resultant membranes were neutralized in $\mathrm{NaOH} 0.1 \mathrm{M}$ solution for $10 \mathrm{~min}$, washed thoroughly with distilled water, and dried again. Samples were labeled according to their cross-linking degree as $\operatorname{CTS}[x(\%)]$. For instance, samples with $x$ $=0.1,1$, and $20 \%$ were labeled as CTS0.1, CTS01, and CTS20, respectively.

Fourier Transform Infrared Spectroscopy with Attenuated Total Reflection (FTIR -ATR). Membranes of both noncross-linked chitosan (CTS00) and cross-linked at the highest ratio (CTS20) were analyzed by infrared spectroscopy to assess the proposed mechanisms for the reaction of chitosan with glutaraldehyde, which may depend on the reaction conditions. ${ }^{9,25,26}$ Spectra were recorded in an IRPrestige 21 FTIR spectrophotometer from Shimadzu (Kyoto, Japan) with the attenuated total reflection accessory (128 scans, resolution $\left.4 \mathrm{~cm}^{-1}\right)$.

X-ray Diffraction (XRD). The morphology of the membranes was analyzed by recording the wide-angle X-ray scattering (WAXS) pattern in a Philips PW1710 reflection diffractometer (Almelo, The Netherlands), with a step $\left(2 \theta=0.02^{\circ}\right)$ scanning time of $2 \mathrm{~s}$ and $\mathrm{Cu} \mathrm{K \alpha}-$ radiation generated at $40 \mathrm{kV}$ and $30 \mathrm{~mA}$.

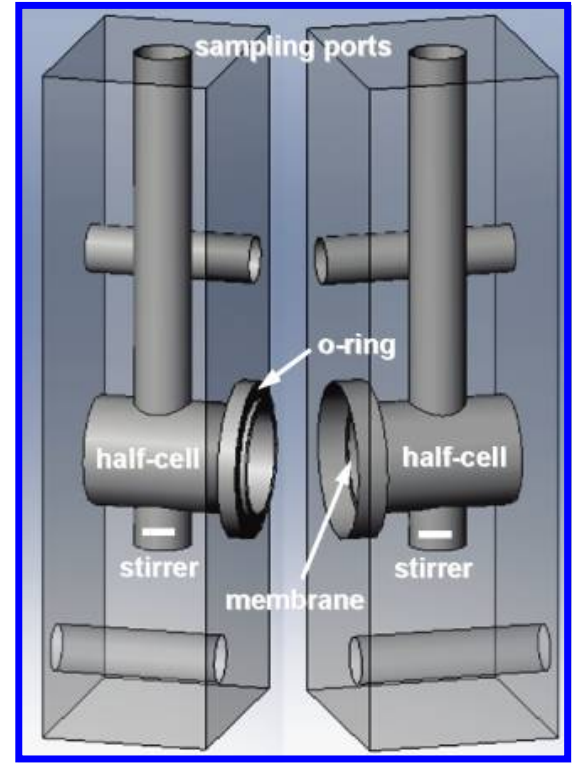

Figure 1. Schematic representation of the in-house built side-by-side diffusion cell.

Water Contact Angle Measurements. The influence of the glutaraldehyde cross-linking on the hydrophilicity of the materials was assessed by evaluating the surface wettability by water contact angle measurements. Static contact angle measurements were carried out by the sessile drop method using a contact angle meter (model OCA 15+) with a high-performance image processing system from DataPhysics Instruments (Filderstadt, Germany). A drop ( $1 \mu \mathrm{L})$ of water was added by a motor driven syringe at room temperature. Two different samples of each material were used and at least three measurements were carried out for each sample.

Equilibrium Swelling Studies. The water uptake measurements were performed in two different buffer systems, both at $\mathrm{pH} 7.4$ and ionic strength $0.154 \mathrm{M}$. Phosphate buffer saline (PBS) solution was prepared by dissolving PBS tablets from Sigma in a suitable amount of water ( $\mathrm{NaCl} 0.137 \mathrm{M} ; \mathrm{KCl} 0.0022 \mathrm{M}$; phosphate buffer $0.01 \mathrm{M} ; \mathrm{pH}$ 7.4 at $25^{\circ} \mathrm{C}$ ). A second solution (TRIS- $\mathrm{Ca}^{2+} / \mathrm{Mg}^{2+}$ ) was prepared with $1.5 \mathrm{mM}$ of $\mathrm{MgCl}_{2}$ and $2.5 \mathrm{mM} \mathrm{CaCl}_{2}$ to yield the typical $\mathrm{Ca}^{2+}$ and $\mathrm{Mg}^{2+}$ concentrations of these cations in the human blood plasma. ${ }^{13}$ This solution was buffered with $0.01 \mathrm{M}$ TRIS and adding $\mathrm{HCl}$ until the $\mathrm{pH}$ was $7.40 \pm 0.05$ at $25^{\circ} \mathrm{C}$. Ionic strength was corrected with $\mathrm{NaCl}$ to give $0.154 \mathrm{M}$.

The equilibrium water uptake was determined by immersing previously weighed chitosan membranes in these buffer systems at $37 \pm$ $1{ }^{\circ} \mathrm{C}$. We previously found that the water uptake kinetics are very fast, reaching equilibrium in less than $15 \mathrm{~min}^{3}$ After around $4 \mathrm{~h}$, equilibrated samples were blotted with filter paper to remove the adsorbed water and weighed immediately. The equilibrium water uptake $\left(\mathrm{WU}_{\mathrm{eq}}\right)$ was calculated using the following equation

$$
\mathrm{WU}_{\mathrm{eq}}=\frac{W-W_{0}}{W_{0}}
$$

where $W_{0}$ is the initial weight of the sample and $W$ is the weight of the swelled sample.

Permeation Studies. The permeation studies were performed using an in-house built side-by-side diffusion cell (see Figure 1). Membranes were previously equilibrated in their respective buffer solution and mounted between the half-cells of the receptor and donor compartments ( $1 \mathrm{~cm}^{2}$ area of diffusion). The receptor compartment fluid was continuously pumped through a flow-through quartz cuvette with an optical pathway $(l)$ of $1 \mathrm{~cm}$. The absorbance at a maximum wavelength (different for each solute) was monitored in a UV-1610 Shimadzu spectrophotometer. The volume of the monitoring system (tubing and quartz cuvette) was calibrated before each experiment and it was found 


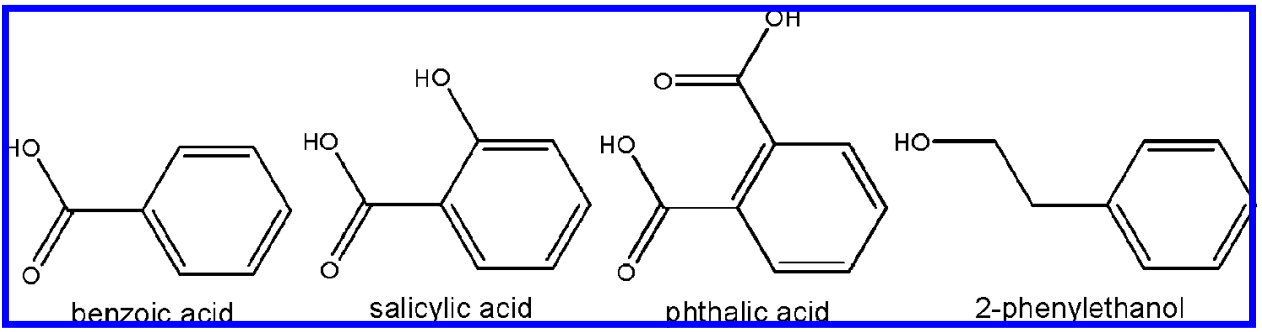

Figure 2. Chemical structures of the model solutes used in the permeation studies.

Table 1. Acidity Constants $\left(p K_{a}\right)$, Wavelength of Maximum UV Absorbance $\left(\lambda_{\max }\right)$, Molar Absorptivity $(\epsilon)$ at $\lambda_{\max }$ for the Different Model Solutes, and Optical Pathway ( $)$ of the Flow-Through Cuvette

\begin{tabular}{lcccc}
\hline \multicolumn{1}{c}{ solute } & $\mathrm{p} K_{\mathrm{a} 1}$ & $\mathrm{p} K_{\mathrm{a} 2}$ & $\lambda_{\max }(\mathrm{nm})$ & $1 /(\epsilon l)(\mathrm{mM})$ \\
\hline benzoic acid (BA) & $4.20^{27}$ & & 270 & 1.747 \\
salicylic acid (SA) & $3.2^{27}$ & & 297 & 0.2816 \\
phthalic acid (Ph) & $2.73^{28}$ & $4.78^{28}$ & 273 & 1.192 \\
2-phenylethanol (PE) & & & 258 & 5.244 \\
\hline
\end{tabular}

to vary between 2.84 and $2.90 \mathrm{~mL}$. The monitoring system was filled with fresh buffer and air bubbles were purged before each measurement. The inlet and outlet tubing was connected to the receptor cell previously filled with $2.2 \mathrm{~mL}$ of fresh buffer. Then, the diffusion cell was immersed in a thermostatic bath at $37.0 \pm 0.1{ }^{\circ} \mathrm{C}$. Finally, the donor cell was filled with $2.2 \mathrm{~mL}$ of the buffer solution containing the respective solute and the absorbance was recorded. The solutions in both cells were stirred by magnetic bars at $800 \mathrm{rpm}$ to eliminate the boundary layer effect. In preliminary experiments, we confirmed that the calculated permeability was kept constant above $400 \mathrm{rpm}$.

The permeability of the chitosan membranes was evaluated for small molecules of similar size but with different chemical moieties, either ionized (anionic) or neutral at physiological pH (see Figure 2). Moreover, solutes with different ionic charges were tested by choosing monoprotic and diprotic acids that are fully ionized at that $\mathrm{pH}$ (see Table 1). The solutions of the anionic solutes were prepared at $5 \mathrm{mM}$ in the case of benzoic acid (BA) and phthalic acid (Ph) or $2 \mathrm{mM}$ for salicylic acid (SA). Because these molecules at working concentration modify the $\mathrm{pH}$ of the buffer solution, this was further corrected to $\mathrm{pH}$ 7.4 with $\mathrm{NaOH}$ solution. The solution of 2-phenylethanol (PE) was prepared at a concentration of $10 \mathrm{mM}$. The different concentrations used took into consideration the different molar absorptivities to allow the detection of the solutes at the early stages of permeation experiments (see Table 1). The flux rate of those model molecules was determined in both PBS and buffered TRIS- $\mathrm{Ca}^{2+} / \mathrm{Mg}^{2+}$ solutions, which was the same used in the water uptake experiments. After each assay, the swollen membrane thickness was taken as a mean of five values at different points measured with a low-pressure micrometer.

Determination of the Partition Coefficients. The partition coefficient $(K)$ was defined as the ratio of the solute concentration in the liquid fraction absorbed by each sample $\left(C_{\mathrm{m}}\right)$ to that in the bulk solution $\left(C_{\mathrm{s}}\right)^{29}$

$$
K=\frac{C_{\mathrm{m}}}{C_{\mathrm{s}}}
$$

$K$ was determined in both PBS and buffered TRIS- $\mathrm{Ca}^{2+} / \mathrm{Mg}^{2+}$ solutions. First the membrane samples were equilibrated for $48 \mathrm{~h}$ in a solution of each model molecule at a concentration $\left(C_{\mathrm{s}}\right) 5$ times higher than that used in the permeation experiments. The $\mathrm{pH}$ of the buffer solutions was corrected to $\mathrm{pH} 7.4$ with $\mathrm{NaOH}$ solution after the solubilization of each acid solute. When compared with the amount of membrane sample, the volume of the concentrated solution was higher enough to consider that $C_{\mathrm{s}}$ did not vary during the solute uptake stage. The membranes were removed from the concentrated solution and the excess liquid was removed by blotting the membrane with filter paper.

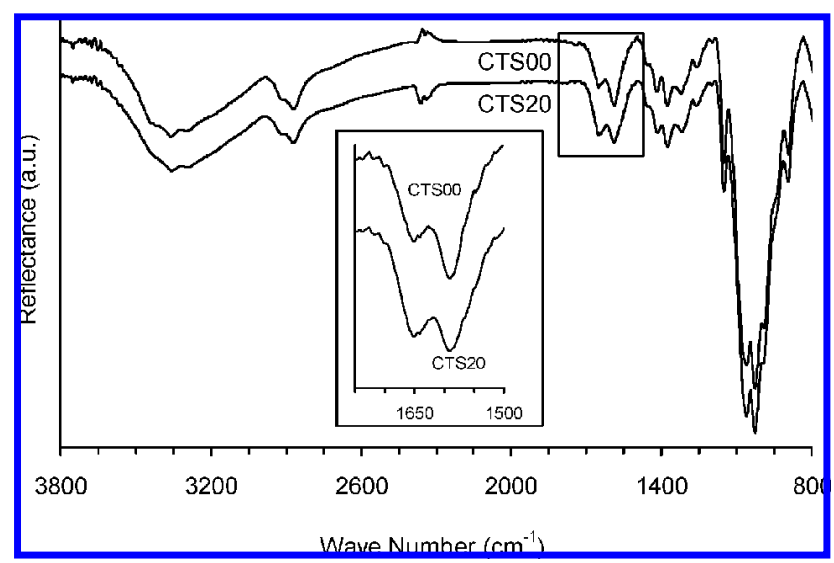

Figure 3. FTIR-ATR spectra of noncross-linked chitosan membranes (CTS00) and at the highest cross-linking degree (CTS20). The inset graphic is the scale up of the spectral region $1500-1750 \mathrm{~cm}^{-1}$.

The water uptake of each sample was determined as described for the equilibrium water uptake experiments. Loaded membranes were immersed in fresh buffer and this procedure was repeated until no further release was observed by UV spectrophotometry. The amount of the solute uptake was calculated as the total cumulative release and $C_{\mathrm{m}}$ was determined accordingly.

\section{Results and Discussion}

Analysis of the Cross-Linking Reaction. Several mechanisms have been proposed for the reaction of chitosan with glutaraldehyde, with the simplest involving the formation of one Schiff base between one of the aldehyde groups and an amine group of chitosan, leaving the other aldehyde group free. ${ }^{9,26}$ This aldehyde group may react with other chitosan chains to form a cross-link but can also remain free, immobilized within the formed polymer network. ${ }^{26}$ A third mechanism may encompass the polymerization of glutaraldehyde, which would form longer cross-linking bridges. ${ }^{9,26}$ Roberts et al. ${ }^{25}$ found that under several reaction conditions only a very small proportion $(<0.15 \%)$ of the aldehyde groups undergo an aldol condensation reaction leading to $\alpha, \beta$-unsaturated aldehyde groups. In turn, Monteiro et al. ${ }^{9}$ found evidence of double ethylenic bonds above a certain glutaraldehyde/chitosan proportion. In our study, the ratio $\mathrm{CHO} / \mathrm{NH}_{2}$ should be low enough to keep the glutaraldehyde polymerization at a very small proportion. The infrared spectrum of the membranes with the highest cross-linking degree (CTS20) is quite similar to the spectrum of the noncross-linked membrane (CTS00; Figure 3). In the chitosan spectrum, the band at $1651 \mathrm{~cm}^{-1}$ (amide I) is assigned to the remaining acetyl groups of chitosan, and the $\mathrm{NH}_{2}$ characteristic absorption band is observed at $1591 \mathrm{~cm}^{-1}$ (amide II). In the spectrum of CTS20 the amide I band increases proportionally to the amide II band. The contribution of the imine $(\mathrm{C}=\mathrm{N})$ bond at around 1660 $\mathrm{cm}^{-19,26}$ from the formed Schiff bases upon cross-linking 
Table 2. Water Contact Angle of Noncross-Linked Chitosan Membranes (CTSO0) and at the Highest Cross-Linking Degree $(\text { CTS20 })^{a}$

\begin{tabular}{cc}
\hline sample & contact angle $\left({ }^{\circ}\right)$ \\
\hline CTS00 & $93.2 \pm 2.1$ \\
CTS20 & $91.0 \pm 0.9$ \\
\hline
\end{tabular}

${ }^{a}$ Average \pm standard deviation.

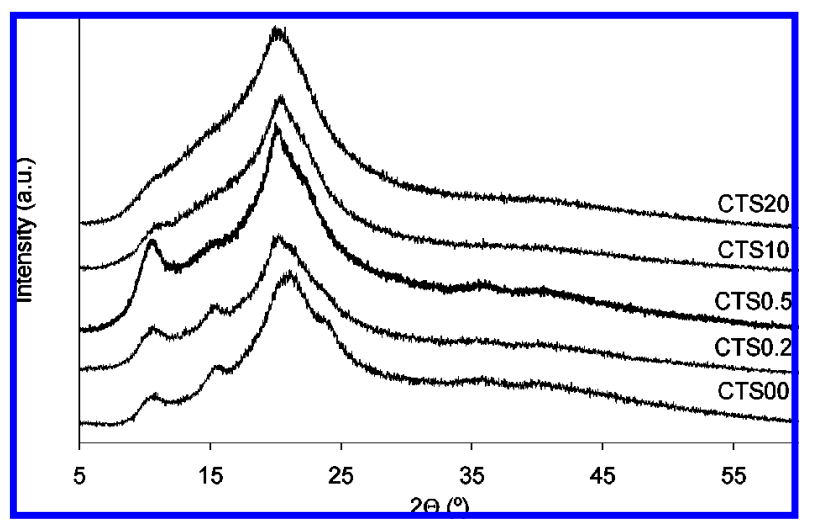

Figure 4. WAXS diffraction patterns for chitosan membranes with cross-linking degrees ranging from 0 up to $20 \%$.

superimposes the amide I band of chitosan, leading to that change in the spectrum.

On the other hand, the signal of the carbonyl groups was not observed at around $1720-1730 \mathrm{~cm}^{-1}$. Because in this region the spectrum of chitosan did not present any absorption band, this constitutes a good indication that, under these homogeneous reaction conditions, most of the glutaraldehyde molecules established a cross-link between chitosan chains being involved in the formation of two Schiff bases. It should be noted that under heterogeneous reaction conditions the appearance of this $\mathrm{CHO}$ band has been reported. ${ }^{26}$ Finally, the water contact angle almost did not change with the reaction with glutaraldehyde molecules (Table 2), indicating that the overall hydrophilicity of the membranes was unaffected.

Morphological Characterization of Chitosan Membranes. Chitosan is a semicrystalline polymer from which several polymorphs have been mentioned in the literature. ${ }^{30-32}$ Chitosan molecular weight, ${ }^{31} \mathrm{DD},{ }^{33}$ and different membrane processing methods gave origin to substantial variations in the presence and amounts of the different polymorphs. ${ }^{31,32}$

Samuels ${ }^{30}$ reported that chitosan has two distinct crystal forms, both orthorhombic. The crystal type I has the strongest reflection at $2 \theta=11.4^{\circ}$, whereas the type II crystal has the strongest reflection, which falls at $2 \theta=20.1^{\circ}$. As can be observed in Figure 4, the noncross-linked chitosan membranes (CTS00) showed the strongest reflection at $2 \theta \approx 20^{\circ}$ and a weaker reflection at $2 \theta \approx 10^{\circ}$, according to the typical chitosan diffractogram. Moreover, it was also possible to observe a reflection at around $2 \theta \approx 15^{\circ}$. Ogawa et al. ${ }^{31,32}$ described that annealed chitosan membranes cast from acetic acid and neutralized with $\mathrm{NaOH}$ may also present an anhydrous crystal form exhibiting a strong reflection at $2 \theta \approx 15^{\circ}$. The cross-linking of chitosan under homogeneous conditions produces a gradual effect on the WAXS patterns manifested by a decrease in the reflection at $2 \theta \approx 15^{\circ}$ already noticed at low degrees of crosslinking (CTS0.5) and the weakening and disappearance of reflections at $2 \theta \approx 10^{\circ}$ and $2 \theta \approx 15^{\circ}$ on approaching the maximum cross-linking degree (CTS20). Surprisingly, the reflection at $2 \theta \approx 20^{\circ}$ related to the type II crystal did not disappear, showing that the chitosan membranes retain the

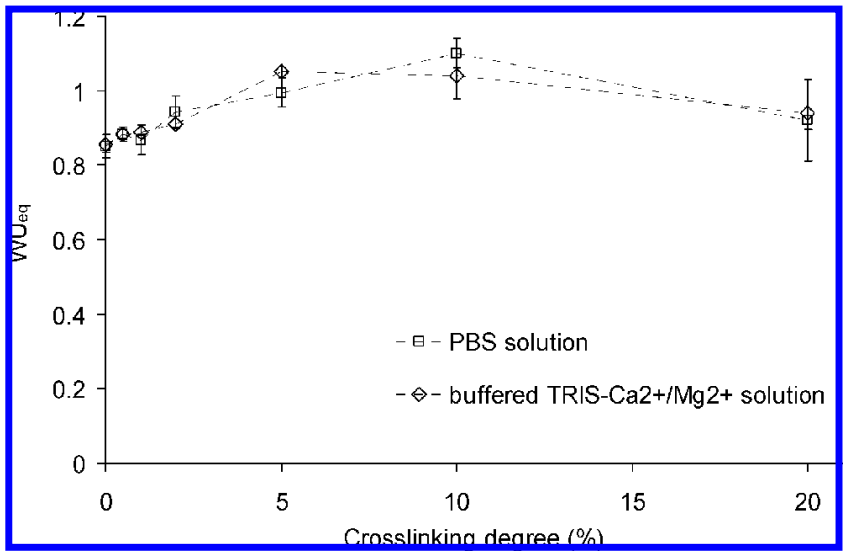

Figure 5. Water uptake (eq 4) of chitosan membranes as a function of the cross-linking degree, either determined in PBS solution or in buffered TRIS-Ca ${ }^{2+} / \mathrm{Mg}^{2+}$ solution. Data represents mean \pm standard deviation for $n=3$.

semicrystalline morphology up to a cross-linking degree of $20 \%$. These results do not agree with our previous findings ${ }^{3}$ that crystallinity was highly depressed for lower cross-linking degrees and that for higher degrees membranes were completely amorphous. Nevertheless, those membranes had been prepared from chitosan with lower $\mathrm{DD},{ }^{3}$ which can explain the difference in the results.

Chitosan Membranes' Equilibrium Swelling Degree and the Influence of Cross-Linking. The chitosan membranes' equilibrium water uptake increased steadily until a cross-linking degree of approximately 10\% (CTS10), opposite to what would be expected (see Figure 5). It is well-documented that the higher the cross-linking degree of a hydrogel, the lower its swelling ratio is. Cross-linking reduces the average molecular weight between cross-links and the mesh size, imposing restrictions to the entrance of water molecules and to the polymer chains relaxation. $^{34,35}$ In fact, a further increase in the cross-linking degree of the chitosan membranes was effective in lowering the equilibrium water uptake.

This atypical behavior of the chitosan membranes at low range cross-linking degrees has already been described in one of our previous works in which membranes were prepared using chitosan with lower DD. ${ }^{3}$ That result has been well-correlated with a sharp reduction in the crystallinity degree already noticeable for faintly cross-linked membranes. ${ }^{3}$ In the herein reported results, the decrease in the crystallinity, which is only related to the disappearance of reflections at $2 \theta \approx 10^{\circ}$ and $2 \theta$ $\approx 15^{\circ}$ (Figure 4 ), can also explain the increase in the equilibrium water uptake shown in Figure 5. Chitosan chains possess a large number of chemical moieties able to undergo hydrogen bonding, and so it is reasonable to consider that a certain number of hydrogen bonds may be established between polymer chains in the amorphous regions in a disorderly fashion. It is also reasonable to consider that the absorbed water in the swollen state did not disrupt all these physical cross-links. Water sorption by polysaccharides has been described by a two-step mechanism: ${ }^{36,37}$ water sorption on polymer-specific sites in the first step and water clustering around the first sorbed water molecules in a second step. Similar hydration behavior has been reported for several neutral polysaccharides. ${ }^{36,37}$ At low water activities, sorption in chitosan membranes occurs on polymer-specific sites until a limit of two water molecules per repeating unit in the amorphous phase. In the second step, retention of four water molecules per chitosan repeating unit is observed, but no further hydrogen bonding is established between water and chitosan 


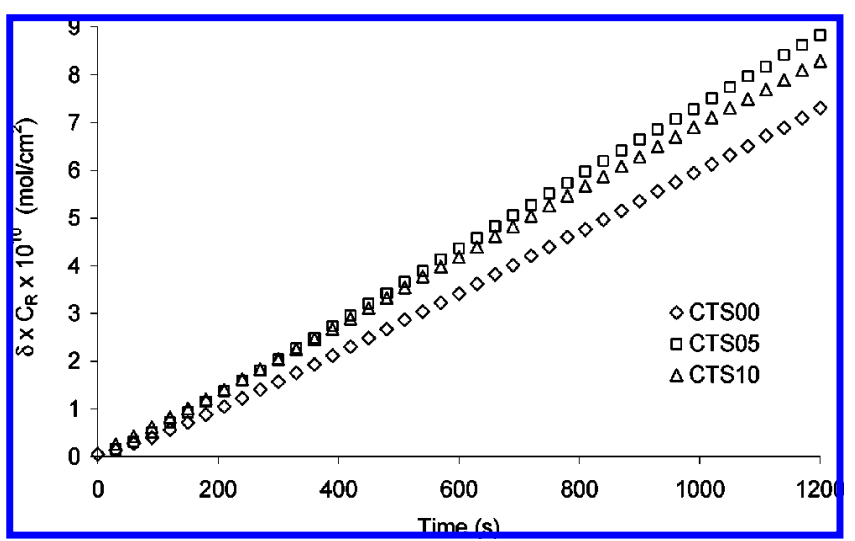

Figure 6. Typical curves of the variation of the concentration $\left(C_{\mathrm{B}}\right)$ of salicylic acid in the receptor compartment normalized by the membrane thickness $(\delta)$.

molecules. ${ }^{36}$ Water molecules may bind both on hydroxyl and on amino groups, ${ }^{38}$ which suggests that those polymer-specific sites are the pyranosyl side substituents and confirms that not all of these groups that are able to hydrogen bond are available to bind water molecules. Being so, the introduction of small amounts of a cross-linker produces steric hindrance, which can reduce the ability of the polymer to establish intermolecular hydrogen bonds and enhance the water molecules accessibility. The hydration water uptake should depend, simultaneously, on the balance between crystallinity, hydrogen bonding between chitosan chains in the amorphous phase, and the cross-linking degree.

Finally, equilibrium water uptake was insensitive to the presence of $\mathrm{Ca}^{2+}$ and $\mathrm{Mg}^{2+}$ in the buffer solution. The wellknown ability of chitosan to form complexes with divalent cations ${ }^{10,11}$ did not influence the water uptake ability, at least at the physiological concentrations of calcium and magnesium.

Permeability of Chitosan Membranes. Typical curves of the early permeation stage of the studied drugs through chitosan membranes are shown in Figure 6, normalized by the membrane thickness, $\delta$, which shows a linear profile without a measurable lag time. This means that flux is constant within that time interval and that the sink conditions are maintained, this is, the concentration in the donor half-cell, $C_{\mathrm{D}}$, is considerably higher than the concentration in the receptor half-cell, $C_{\mathrm{R}},\left(C_{\mathrm{D}}-C_{\mathrm{R}}\right.$ $\approx C_{\mathrm{D}}$ ). The slope of the permeation curves was estimated by linear regression at that early release stage (correlation factor $>0.99$ ).

The permeability, $P$, was calculated using the following equation

$$
P=\frac{V_{\mathrm{R}}}{A}\left(\frac{\mathrm{d} C_{\mathrm{R}}}{\mathrm{d} t}\right) \frac{\delta}{C_{\mathrm{D}}}
$$

where $V_{\mathrm{R}}$ is the volume of the receptor half-cell (including the volume of the UV spectrophotometry monitoring system) and $A$ is the membrane useful mass transfer area.

The apparent diffusion coefficients $(D)$ were determined using eq 7 , considering the propagation of random errors associated with the experimental measurement of $K$ and $P$.

$$
D=P / K
$$

In Figure 7 it is possible to observe the values of $D, K$, and $P$ for permeation of the different solutes through noncross-linked chitosan membranes (CTSO0). The permeability of the different model solutes (see Figure 7a) increased according to the following sequence: $\mathrm{SA}>\mathrm{PE}>\mathrm{Ph} \sim \mathrm{BA}$. The great difference

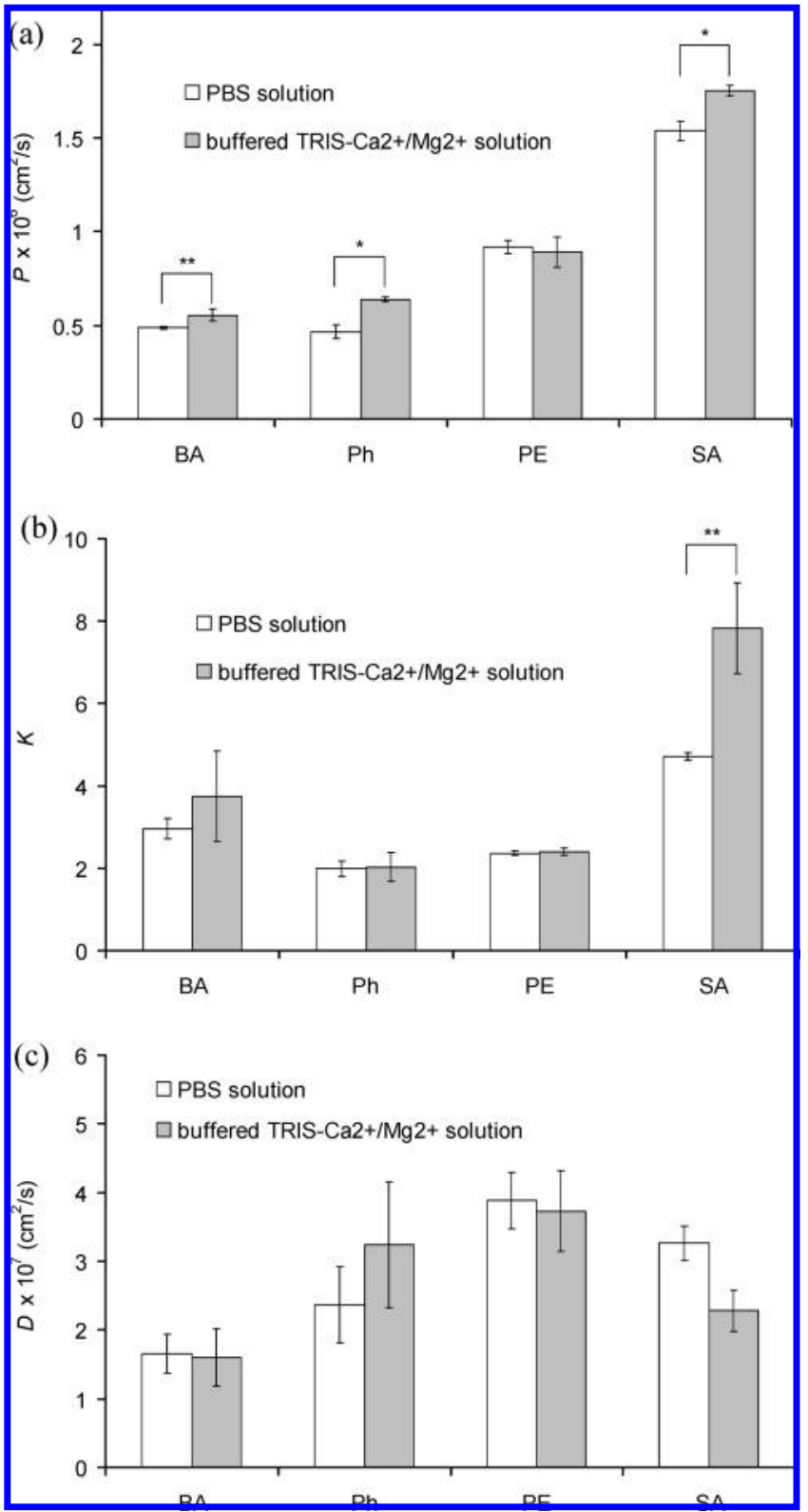

Figure 7. Permeability (a), partition (b), and apparent diffusion (c) coefficients of benzoic acid (BA), salicylic acid (SA), phthalic acid (Ph), and 2-phenylethanol (PE), determined for chitosan membranes (CTS00) in either PBS solution or in buffered TRIS- $\mathrm{Ca}^{2+} / \mathrm{Mg}^{2+}$ solution. Data represents mean \pm standard deviation for $n=3$ ( $^{*} p<$ $\left.0.01 ;{ }^{* *} p<0.05\right)$.

in the permeability of salicylic acid with respect to the other molecules is mainly due to its higher affinity to the chitosan matrix, as confirmed by its much higher partition coefficient (see Figure 7b). Chitosan membranes do not hold charged amine groups at the physiological $\mathrm{pH},{ }^{39}$ thus electrostatic interactions are not expected to occur directly with charged anionic solutes. The affinity of the model solutes to the membrane should be related to other type of interactions. Chitosan has been extensively studied for its cation binding capacity in neutral or weakly acid media. ${ }^{12}$ The mechanism of coordination involved in the formation of complexes is still controversial, but it is generally accepted that the amine groups are the complexation sites of chitosan. The coordination bonds should be established between the free electron pairs of the coordinate (amine group) and the void orbitals of the metal. ${ }^{10}$ Although chitosan has been reported to present a better ability to chelate ions of transition 

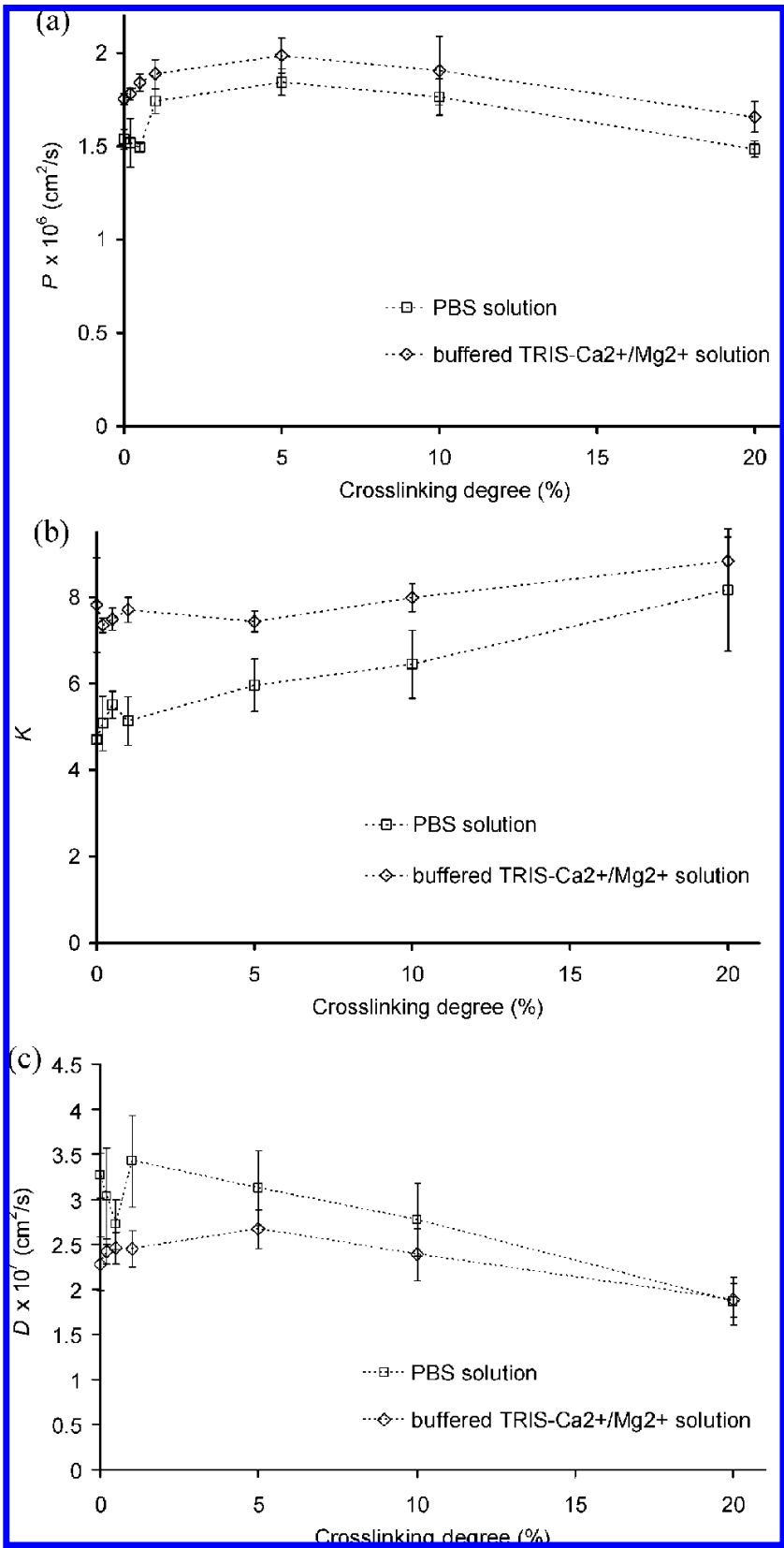

Figure 8. Permeability (a), partition (b), and apparent diffusion (c) coefficients of salicylic acid determined in either PBS solution or in buffered TRIS- $\mathrm{Ca}^{2+} / \mathrm{Mg}^{2+}$ solution as a function of the membranes cross-linking degree. Data represents mean \pm standard deviation ( $n$ $=3)$.

metals, sorption has also been observed for alkaline-earth metal cations. ${ }^{10,11}$ Interestingly, the buffer system that included $\mathrm{Ca}^{2+}$ and $\mathrm{Mg}^{2+}$ at the typical concentrations in the human blood plasma induced an increase in the permeability of the anionic solutes ( $\mathrm{SA}, \mathrm{Ph}$, and $\mathrm{BA}$ ) but did not have a statistically significant effect on the neutral one (PE; Figure 7a). In the case of SA, the increase in the permeability in the presence of divalent ions can be again correlated with the increase in the partition coefficient.

The side-by-side diffusion cell was also used to evaluate the transport properties of the low molecular weight molecules through chitosan membranes with different cross-linking degrees. The SA was chosen to evaluate the effect of cross-linking in the permeation properties of chitosan membranes (see Figure 8).
The permeability of chitosan membranes to the SA followed the same tendency of the equilibrium water uptake, increasing until a cross-linking degree of approximately 5\% (CTS05) and then decreasing steadily until $20 \%$ (Figure $8 \mathrm{a}$ ). The permeability measured in the presence of $\mathrm{Ca}^{2+}$ and $\mathrm{Mg}^{2+}$ in the buffer solution followed the same tendency but it presented higher values for each sample. The difference between the permeability measured either in the presence or in the absence of those cations is almost the same throughout the cross-linking degree scale. The apparent diffusion coefficient showed a similar dependence on the cross-linking degree, but the difference between the two buffer solution groups decreased with the cross-linking degree, converging for higher cross-linking degrees (Figure 8c). On the other hand, the partition coefficient of the SA increased almost linearly with the cross-linking degree from 0 up to $20 \%$. It is interesting to notice that the partition coefficients measured in the different buffers also seem to converge to the same value as the cross-linking degree increases (Figure 8b). This result gives a strong indication that the higher affinity of SA to the chitosan membranes in the presence of $\mathrm{Ca}^{2+}$ and $\mathrm{Mg}^{2+}$ at the physiological concentration is directly related to the complexation of these divalent cations with chitosan. In fact, it has been reported that cross-linking chitosan with glutaraldehyde decreases $\mathrm{Cu}^{2+}$ binding capacity. ${ }^{9}$

This should not be surprising because the several mechanisms proposed for the complex formation involve bonding of the metal ion to one or several amine groups, ${ }^{10,12}$ whose availability decreases as the cross-linking degree increases. The change in the permeation properties of chitosan to anionic solutes in the presence of these metallic cations is an important result and should be taken into consideration in the in vitro predictions of the drug release from chitosan-based controlled drug release systems. Moreover, the influence of these divalent cations on the permeation properties of chitosan, if generalized to other polyelectrolytes able to form ionic complexes, can also raise concerns on how the ionic environment at physiological conditions will affect the final performance of controlled drug release devices based on these polyelectrolytes.

\section{Conclusions}

Although the cross-linking of chitosan under homogeneous conditions produces a gradual effect on the WAXS patterns, manifested by a weakening and disappearance of reflections at $2 \theta \approx 10^{\circ}$ and $2 \theta \approx 15^{\circ}$, the reflection at $2 \theta \approx 20^{\circ}$ related to the type II crystal did not disappear, showing that the chitosan membranes retain the semicrystalline morphology up to a crosslinking degree of $20 \%$.

The equilibrium water uptake and the permeability of chitosan membranes followed a similar tendency, increasing until an intermediate cross-linking degree (10 and 5\%, respectively) and then decreasing. The buffer system that included $\mathrm{Ca}^{2+}$ and $\mathrm{Mg}^{2+}$ at the typical human blood plasma concentrations induced a statistically significant increase in the permeability of the anionic solutes but did not have any effect on the neutral one. The higher affinity of SA to the chitosan membranes in the presence of $\mathrm{Ca}^{2+}$ and $\mathrm{Mg}^{2+}$ at the physiological concentration seems to be directly related to the complexation of these divalent cations with chitosan. The permeation properties of chitosan to anionic solutes, depending on the presence of such metallic cations, is an important result, which should be taken into consideration when trying to make in vitro predictions of the drug release from chitosan-based controlled release systems. We also hypothesize that a similar effect of these metallic cations can 
also be found in hydrogels prepared from other polyelectrolyte sources able to form ion-polymer complexes.

Acknowledgment. This work was partially supported by the Portuguese Foundation for Science and Technology (FCT), through funds from the POCTI and FEDER programmes, and through the scholarship SFRH/BD/6862/2001 granted to R.M.P.d.S. This work was carried out under the scope of the European NoE EXPERTISSUES (NMP3-CT-2004-500283) and was also partially supported by the EU funded projects HIPPOCRATES (STREP-NMP3-CT-2003-505758) and PROTEUS (INTERREG III A-SP1.P151/03).

Supporting Information Available. A detailed description of the equation deduction used to calculate the permeability from the experimental data. This information is available free of charge via the Internet at http://pubs.acs.org.

\section{References and Notes}

(1) Prabaharan, M.; Mano, J. F. Drug Delivery 2005, 12, 41-57.

(2) Thacharodi, D.; Panduranga Rao, K. Biomaterials 1996, 17, 1307.

(3) Silva, R. M.; Silva, G. A.; Coutinho, O. P.; Mano, J. F.; Reis, R. L. J. Mater. Sci.: Mater. Med. 2004, 15, 1105-1112.

(4) Lopez-Perez, P. M.; Marques, A. P.; da Silva, R. M. P.; Pashkuleva, I.; Reis, R. L. J. Mater. Chem. 2007, 17, 4064-4071.

(5) Tuzlakoglu, K.; Alves, C. M.; Mano, J. F.; Reis, R. L. Macromol. Biosci. 2004, 4, 811-819.

(6) Silva, G. A.; Ducheyne, P.; Reis, R. L. J. Tissue Eng. Regen. Med. 2007, 1, 4-24.

(7) Finkenzeller, G.; Torio-Padron, N.; Momeni, A.; Mehlhorn, A. T.; Stark, G. B. Tissue Eng. 2007, 13, 1413-1420.

(8) Laschke, M. W.; Harder, Y.; Amon, M.; Martin, I.; Farhadi, J.; Ring, A.; Torio-Padron, N.; Schramm, R.; Rucker, M.; Junker, D.; Haufel, J. M.; Carvalho, C.; Heberer, M.; Germann, G.; Vollmar, B.; Menger, M. D. Tissue Eng. 2006, 12, 2093-2104.

(9) Monteiro, O. A. C.; Airoldi, C. Int. J. Biol. Macromol. 1999, 26, 119128.

(10) Rhazi, M.; Desbrieres, J.; Tolaimate, A.; Rinaudo, M.; Vottero, P.; Alagui, A.; El Meray, M. Eur. Polym. J. 2002, 38, 1523-1530.

(11) Bravo-Osuna, I.; Millotti, G.; Vauthier, C.; Ponchel, G. Int. J. Pharm. 2007, 338, 284.

(12) Guibal, E. Sep. Purif. Technol. 2004, 38, 43-74.

(13) Oyane, A.; Kim, H. M.; Furuya, T.; Kokubo, T.; Miyazaki, T.; Nakamura, T. J. Biomed. Mater. Res., Part A 2003, 65A, 188-195.

(14) Braccini, I.; Grasso, R. P.; Perez, S. Carbohydr. Res. 1999, 317, 119130 .
(15) Backfolk, K.; Lagerge, S.; Rosenholm, J. B.; Eklund, D. J. Colloid Interface Sci. 2002, 248, 5-12.

(16) Debon, S. J. J.; Tester, R. F. Food Chem. 2001, 73, 401-410.

(17) Han, S. C.; Choo, K. H.; Choi, S. J.; Benjamin, M. M. J. Membr. Sci. 2007, 290, 55-61.

(18) Konradi, R.; Ruhe, J. Macromolecules 2004, 37, 6954-6961.

(19) Navarro, R. R.; Wada, S.; Tatsumi, K. J. Hazard. Mater. 2005, 123, 203-209.

(20) Murdan, S. J. Controlled Release 2003, 92, 1-17.

(21) Cafaggi, S.; Russo, E.; Stefani, R.; Leardi, R.; Caviglioli, G.; Parodi, B.; Bignardi, G.; De Totero, D.; Aiello, C.; Viale, M. J. Controlled Release 2007, 121, 110-123.

(22) Liu, D. Z.; Sheu, M. T.; Chen, C. H.; Yang, Y. R.; Ho, H. O. J. Controlled Release 2007, 118, 333-339.

(23) Terbojevich, M.; Cosani, A.; Muzzarelli, R. A. A. Carbohvdr. Polvm. 1996, 29, 63-68.

(24) da Silva, R.; Mano, J.; Reis, R. Macromol. Chem. Phys. 2008, 209, Doi: 10.1002/macp.200800191.

(25) Roberts, G. A. F.; Taylor, K. E. Die Makromol. Chem. 1989, 190, 951-960.

(26) Tual, C.; Espuche, E.; Escoubes, M.; Domard, A. J. Polvm. Sci., Part B: Polvm. Phys. 2000, 38, 1521-1529.

(27) Robinson, D.; Smith, J. N.; Williams, R. T. Biochem. J. 1953, 55, $151-155$.

(28) Gluck, S. J.; Steele, K. P.; Benko, M. H. J. Chromatogr., A 1996, 745, 117-125.

(29) Matsuyama, H.; Kitamura, Y.; Naramura, Y. J. Appl. Polvm. Sci. 1999, 72, 397-404.

(30) Samuels, R. J. J. Polym. Sci., Part B: Polym. Phys. 1981, 19, 10811105.

(31) Ogawa, K.; Yui, T.; Miya, M. Biosci. Biotechnol. Biochem. 1992, 56, $858-862$.

(32) Ogawa, K.; Hirano, S.; Miyanishi, T.; Yui, T.; Watanabe, T. Macromolecules 1984, 17, 973-975.

(33) Zhang, Y. Q.; Xue, C. H.; Xue, Y.; Gao, R. C.; Zhang, X. L. Carbohydr. Res. 2005, 340, 1914-1917.

(34) Stringer, J. L.; Peppas, N. A. J. Controlled Release 1996, 42, 195202.

(35) Amende, M. T.; Hariharan, D.; Peppas, N. A. React. Polym. 1995, 25, 127-137.

(36) Despond, S.; Espuche, E.; Cartier, N.; Domard, A. J. Polvm. Sci., Part B: Polvm. Phys. 2005, 43, 48-58.

(37) Fringant, C.; Desbrieres, J.; Milas, M.; Rinaudo, M.; Joly, C.; Escoubes, M. Int. J. Biol. Macromol. 1996, 18, 281-286.

(38) Rueda, D. R.; Secall, T.; Bayer, R. K. Carbohydr. Polvm. 1999, 40, 49-56.

(39) Wang, Q. Z.; Chen, X. G.; Liu, N.; Wang, S. X.; Liu, C. S.; Meng, X. H.; Liu, C. G. Carbohydr. Polvm. 2006, 65, 194-201.

BM8001649 